\title{
Comparison of the combustion behaviours of agricultural wastes under dry air and oxygen
}

\author{
H. Haykiri-Acma \& S. Yaman \\ Istanbul Technical University, Department of Chemical Engineering, \\ Istanbul, Turkey
}

\begin{abstract}
Biomass is a renewable fuel and the fourth largest energy source following coal, oil, and natural gas. Besides, agricultural wastes account for an important part of biomass, and they have not been evaluated properly. Although some conversion methods have been also applied to biomass to produce pyrolytic liquids, gaseous fuels or activated carbon, combustion is still the most applicable method to take advantage of the energy potential of biomass. In this study, some agricultural waste biomass materials such as sunflower seed shell (SSS), hazelnut shell (HS), rice hull $(\mathrm{RH})$, and olive husk $(\mathrm{OH})$, all of which have great potential in Turkey were regarded as renewable energy sources. In order to compare the combustion reactivities of these materials, burning tests were carried out under different atmospheres such as dry air and oxygen. For this purpose, biomass materials were burned in a thermal analyser to obtain TGA (Thermogravimetric Analysis) and DSC (Differential Scanning Calorimetry) thermograms under both conditions. Initial sample mass was approximately $10 \mathrm{mg}$ for each sample which has a particle size of $<0.25 \mathrm{~mm}$, and temperature was raised from ambient to $900^{\circ} \mathrm{C}$ with a heating rate of $40^{\circ} \mathrm{C} / \mathrm{min}$ under gas flow rate of $100 \mathrm{~mL} / \mathrm{min}$. No hold time was allowed at the final temperature. This study showed that the thermal reactivities of biomass species change in a wide region, and the type of the oxidative medium plays very important effects on the burning parameters such as the ignition point, maximum rate of combustion and its temperature as well as the end point of burning. On the other hand, macromolecular ingredients of biomass such as hemicellulosics, celluloses, and lignin have significant effects on the combustion behaviour of these waste materials.

Keywords: biomass, agricultural wastes, combustion, dry air, oxygen.
\end{abstract}




\section{Introduction}

Turkey is a country that imports the most part of its energy need, so the foreign dependency on energy is steadily increasing. Although the fossil energy sources provide the significant portion of energy need in this country, national oil and natural gas reserves are incapable of meeting the need. On the other hand, a great deal of coal reserves in Turkey is comprised of lignites which have low calorific value and high mineral matter.

On the other hand, Turkey has a big potential of biomass energy resources. Biomass is regarded as renewable, carbondioxide-free, environmental friendly, sustainable, and alternative energy resource which can be easily found in all parts of the world (Klass [1]). Any organic substance except fossil fuels is defined as biomass which is mainly consisted of carbon, hydrogen, and oxygen, and it is generally comprised of agricultural-, industrial-, and municipal solidwastes, bio-solids, energy crops, forestry remnants, animal wastes, and aquatic flora (Clark and Deswarte [2]). Since, the most of biomass species are accepted as carbondioxide neutral, they show lower environmental impact compared to fossil fuels, and their contribution to the increase in atmospheric carbondioxide concentration is almost negligible. Moreover, biomass energy resources are renewable and low cost, and they have sustainable potential throughout the world. So, it is very important to dispose waste biomass in such a way that their energy potential is used without causing environmental problems.

Although Turkey has a great biomass energy potential, most of the waste biomass species could not be evaluated properly for the energetic purpose. Landfilling that is still the most extensive way to dispose such wastes also causes a couple of ecological problems and environmental pollution such as methane production, contamination of underground water deposits, and other emissions. Thus, landfilling should not be seen an option for disposal of organic remnants that decompose easily, and their energy potential must be evaluated during the disposal method applied.

Although some conversion methods have been also applied to biomass to produce pyrolytic liquids, gaseous fuels or activated carbon, combustion is still the most applicable method to take advantage of the energy potential of biomass (Basu [3], Haykiri-Acma and Yaman [4], Miller and Tillman [5]). In this context, the intrinsic properties of biomass as well as the conditions in the combustion system play critical role from various aspects. In fact, biomass materials are thermally more reactive than most coal samples in general owing to their high oxygen and volatile matter contents. On the other hand, some macromolecular ingredients of biomass such as hemicellulosics and celluloses show very high thermal reactivities during thermal processes. These factors make the combustion of biomass highly complex, and the performance of the combustors is closely affected from these reactivity-related characteristics. From these points of view, the combustion reactivities of some agricultural waste biomass species have been studied in this study. 


\section{Materials and methods}

Agricultural waste biomass resources were provided from the Turkish food industry and they are kept at laboratory medium for 15 days to allow elimination of the free moisture. Then, air-dried samples were milled and screened through a sieve having an opening of $0.25 \mathrm{~mm}$. The proximate analysis and the gross calorific value measurements of the biomasses were conducted according to ASTM standards, and the ultimate analyses were performed by an elemental analyzer (EuroEA3000 model).

Major biomass ingredients in the biomass materials were determined by the following analytical procedures. Extractive components were determined according to ASTM D1105 (2001). The sample remaining after alcohol-benzene extractions was used to find the holocellulosics by means of $\mathrm{NaClO}_{2}$ extraction procedure. The lignin content of the sample was found according to the method of van Soest [6].

Burning profiles (TGA and DSC) of the biomass samples were obtained by TA Instruments SDT Q600 model thermal analyser. For this, around $10 \mathrm{mg}$ of sample was heated from ambient to $900^{\circ} \mathrm{C}$ with a heating rate of $40^{\circ} \mathrm{C} / \mathrm{min}$ under either dry air or pure oxygen flow of $100 \mathrm{~mL} / \mathrm{min}$, and no hold time was allowed at the final temperature.

\section{Results and discussion}

Proximate analysis, ultimate analysis, and gross calorific value results of the parent (untreated) biomass materials are given in Table 1, and it is possible to

Table 1: $\quad$ Analysis results of the samples.

\begin{tabular}{llrrrr}
\hline & & $\begin{array}{c}\text { Sunflower } \\
\text { Seed Shell }\end{array}$ & $\begin{array}{c}\text { Hazelnut } \\
\text { Shell }\end{array}$ & $\begin{array}{c}\text { Olive } \\
\text { Milling } \\
\text { Residue }\end{array}$ & $\begin{array}{c}\text { Rice } \\
\text { Husk }\end{array}$ \\
\cline { 2 - 6 } Proximate & Volatiles & 83.7 & 72.0 & 68.6 & 61.4 \\
Analysis & Fixed Carbon* & 13.6 & 21.0 & 10.4 & 15.4 \\
(\%, dry basis) & Ash & 2.7 & 7.0 & 21.0 & 23.2 \\
\hline Ultimate & $\mathrm{C}$ & 47.8 & 54.8 & 48.3 & 39.6 \\
Analysis & $\mathrm{H}$ & 6.1 & 6.7 & 5.0 & 6.0 \\
(\%, dry-ash-free & $\mathrm{N}$ & 1.2 & 1.0 & 1.2 & 0.7 \\
basis) & $\mathrm{S}$ & 0.3 & 0.1 & 0.0 & 0.0 \\
& $\mathrm{O}$ & 44.6 & 37.4 & 45.5 & 53.7 \\
\hline Structural & Extractives & 13.8 & 6.2 & 13.6 & 9.8 \\
Analysis & Lignin & 31.4 & 51.5 & 34.7 & 34.8 \\
(\%, dry basis) & Holocellulose & 62.5 & 38.6 & 40.0 & 44.9 \\
\hline Calorific & Higher Calorific & & & & \\
Analysis & Value (MJ/kg) & 17.7 & 18.2 & 15.9 & 13.4 \\
(as received) & & & & & \\
\hline
\end{tabular}

* calculated by difference 
conclude that sunflower seed shell is the richest biomass sample with respect to holocellulose (hemicellulosics + cellulose forms) content. It is well known-that the higher the holocellulose content, the higher the thermal reactivity (Klass [1]). On the other hand, the highest lignin content belongs to hazelnut shell, and lignin is not as thermally reactive as holocellulose (Haykiri-Acma and Yaman [7], Haykiri-Acma et al. [8]). Besides, ash contents of the samples change in a wide range, while their hydrogen and nitrogen contents are at the comparable levels. High ash content of rice husk has a reducing influence on the calorific value and consequently the lowest calorific value was detected for rice husk. Oxygen contents of the biomass samples are quite high and this is an indication of the high thermal reactivity (Klass [1]). In fact, high oxygen content is one of the important characteristics of most biomass materials. Besides, fixed carbon contents of the biomass samples are lower than that for coal in general. This explains the relatively low calorific values of the biomasses used in this study. In addition, ash contents of the samples varies in a so wide range that the ash content of SSS is only $2.7 \%$ while the ash content of RH reaches $23.2 \%$. Sulfur contents of the biomass species are very low regarding the ash contents of the low rank coals in general. On the other hand, hydrogen and nitrogen contents of all the samples are very close to each other.

TGA and DSC thermograms of the samples obtained under dry air and oxygen are given in Figures 1 and 2, respectively.

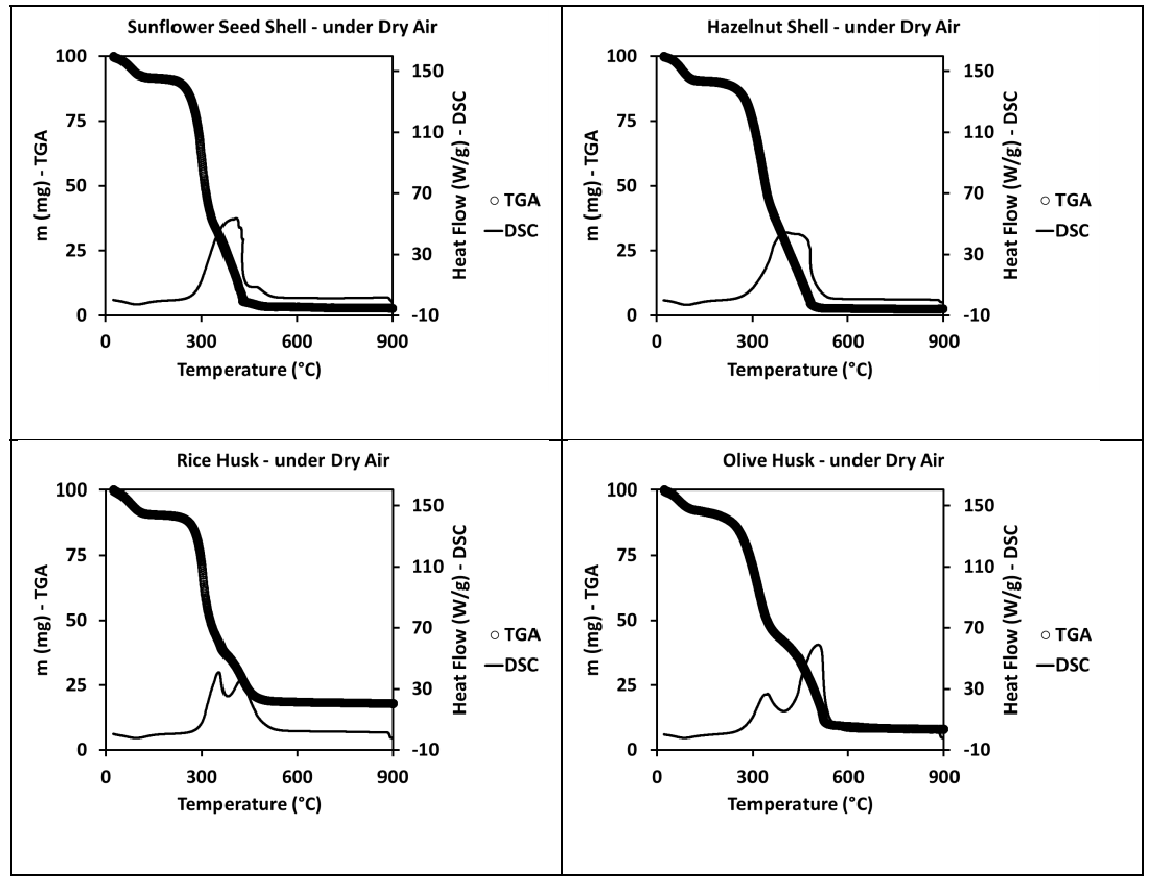

Figure 1: TGA and DSC thermograms under dry air. 


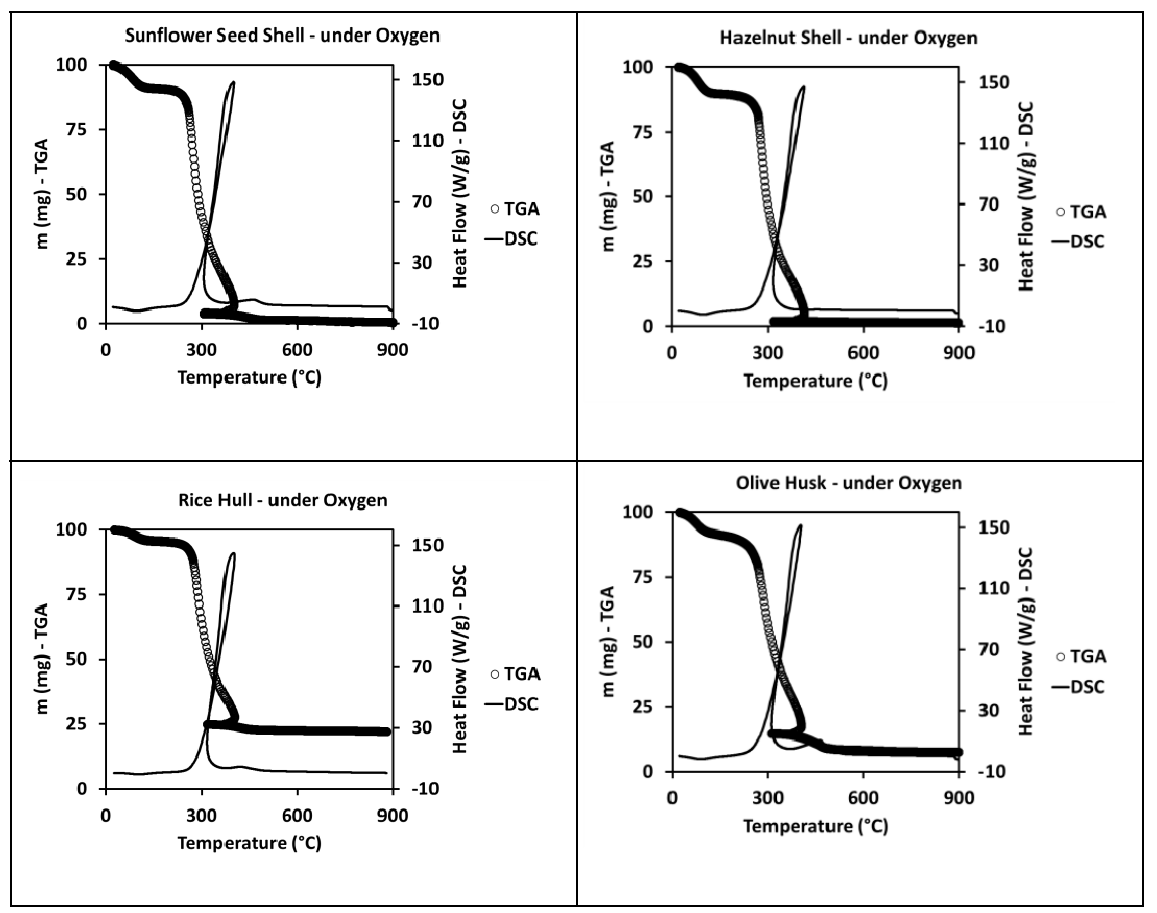

Figure 2: $\quad$ TGA and DSC thermograms under oxygen.

TGA thermograms in Figure 1 indicate that there is a low temperature region in which moisture content is eliminated from the biomass samples. After that relatively more stagnant process takes place in which less amount of solid matter decomposes. At around $250^{\circ} \mathrm{C}$, rapid mass losses are observed, predicting the decomposition of thermally unstable hemicellulosics and partly celluloses. This process is known as the volatilisation, and as a result of this both combustible and non-combustible volatiles form. Whenever temperature is high enough, combustible volatiles ignite under oxidative conditions, and homogeneous combustion in the gaseous phase begins. This process is so rapid that the mass losses which are almost vertical can be distinguished on TGA thermograms of each biomass species. Later, the slope of this trend decreases indicating the start of another process in which combustion is progressed by means of burning of the fixed carbon. The burning of fixed carbon is rather a slow process and it takes longer time in comparison to combustion of volatiles. Finally, the unchanged mass of sample is attained as temperature increases. Comparison of the slopes of the TGA thermograms in the main combustion stages predicts the fact that SSS is the more reactive biomass sample investigated in this study. In fact, the highest volatile matter content as well as the highest holocellulose content belongs to SSS, and the abundance of such constituents makes this biomass very reactive under combustion conditions. On the other hand, SSS and HS that have 
relatively low contents of ash gave very high burn-out performances that almost all of the solid matter could be burned/decomposed under investigated conditions, while significant amounts of residues were produced from $\mathrm{RH}$ and $\mathrm{OH}$ samples.

As to DSC thermograms, it is likely to say that each sample has its own heatgiving characteristics. That is, SSS and HS produced a unique curve under which exothermic heat released. Whereas, RH gave such an exothermic region that there were two maxima at different temperatures. Besides, $\mathrm{OH}$ gave extremely different two exothermic regions which can be attributed to the contribution of volatiles and the fixed carbon to the calorific value. On the other hand, the largest area under the exothermic curve was determined in case of HS which is consistent with the fact that it has the highest calorific value among the samples.

TGA and DSC thermograms in Figure 2 designate that the thermal reactivities and the combustion behaviour of the biomasses are highly different under pure oxygen flow from those under dry air. The most striking difference can be seen in the increase in the combustion reactivity. Consequently, the main combustion region on TGA became so much more vertical that it almost consists of only one step. This shows that it is impossible to say that different mechanisms coexist during the main combustion stage when pure oxygen is used. Interestingly, some temperature drops were determined just after ending of the main combustion stage, and the temperature drops continued until around $300^{\circ} \mathrm{C}$. Then, further mass losses until the final temperature were almost negligible.

DSC thermograms obtained under pure oxygen flow were not in the shape of board peaks as in the case of dry air. Namely, very sharp peaks that are rather in the shape of lines were observed, indicating very high rates of heat flows during burning with pure oxygen. This is a clear indication of the increasing thermal reactivity under oxygen atmosphere. Accordingly, the levels of the maximum heat flow rates changed from the range of 40-60 W/g to $130-150 \mathrm{~W} / \mathrm{g}$ under dry air and oxygen flows, respectively. Moreover, these high rates of heat flows were obtained relatively at lower temperatures. This is also another indication of the increasing reactivity under oxygen flow.

\section{Conclusion}

Combustion reactivities of some biomass species including sunflower seed shell, hazelnut shell, rice hull, and olive husk were investigated using TGA and DSC techniques under dry air and pure oxygen flows. It was concluded that the differences in chemical properties of these biomass materials measured by proximate, elemental, and structural analyses are also evident in the burning properties. Volatile matter-rich and holocellulose-rich samples are likely to be decomposed at low temperatures. Increase in the oxygen content of the oxidizing medium enhanced the combustion reactivities of the samples considerably. 


\section{References}

[1] Klass D.L, Biomass for renewable energy, fuels, and chemicals, San Diego, Academic Press, 1998.

[2] Clark J. and Deswarte F, Introduction to chemicals from biomass, Wiley, 2008.

[3] Basu P., Biomass gasification and pyrolysis. Practical design and theory, Burlington, Elsevier, 2010.

[4] Haykiri-Acma H, Yaman S., Thermal reactivity of rapeseed (Brassica Napus L) under different gas atmospheres, Bioresource Techn., 99, 237-242, 2008.

[5] Miller B.G., Tillman D.A., Combustion engineering issues for solid fuel systems, San Diego, Elsevier, 2008.

[6] Van Soest PJ, Use of detergents in the analysis of fibrous feeds. II. A rapid method for the determination of fiber and lignin, J Assoc Offic Anal Chem, 46, 829-835, 1963.

[7] Haykiri-Acma H., Yaman S, Thermogravimetric investigation on the thermal reactivity of biomass during slow pyrolysis, Int J of Green Energy, 6, 333-342, 2009.

[8] Haykiri-Acma H., Yaman S., Kucukbayrak S., Comparison of the thermal reactivities of isolated lignin and holocellulose during pyrolysis, Fuel Processing Tech., 91, 759-764, 2010. 